Editorial Manager(tm) for Journal of Strain Analysis for Engineering Design

Manuscript Draft

Manuscript Number:

Title: Fracture Mechanics approach to design analysis of notches, steps and internal cut-outs in planar components

Article Type: Paper

Keywords: stress concentration factor; notch interaction; notch shielding; slots; steps; U-grooves

Corresponding Author: Professor Thomas G.F. Gray, Ph.D

Corresponding Author's Institution: University of Strathclyde

First Author: thomas gray, BSc PhD

Order of Authors: thomas gray, BSc PhD; Thomas G.F. Gray, Ph.D

Abstract: A new approach to the assessment and optimisation of geometric stress-concentrating features is proposed, based on the correspondence between sharp crack or corner stress-field-intensity factors and conventional elastic stress concentration factors for radiused transitions. This approach complements the application of finite element analysis and the use of standard SCF data from the literature. The method makes it possible to develop closed form solutions for stress concentration factors, in cases where corresponding solutions for the sharp crack geometries exist. This is helpful in the context of design optimisation. The analytical basis of the correspondence is shown, together with the limits on applicability where stress-free boundaries nearby the stress concentrating feature are present or adjacent features interact. Examples are given which compare parametric results derived from finite element analysis with closed form solutions based on the proposed method. New information is given on the stress state at a $90^{\circ}$ corner or width step, where the magnitude of the stress-field-intensity is related to that of the corresponding crack geometry. This correspondence enables the user to further extend the application of crack-tip stressfield-intensity information to square-cornered steps, external U-grooves and internal cut-outs. 

Covering Letter for Paper by T G.F. Gray and J. Wood

Fracture Mechanics approach to design analysis of notches, steps and internal cut-outs in planar components

Dear Editor,

This paper is intended to provide a useful tool to assist designers of structural and mechanical components containing geometric stress concentrations. It has arisen in part through the first author's long association as a member of the Stress Analysis and Strength of Components Committee of the Engineering Sciences Data Unit and its collection of Data Items on elastic stress concentration factors in a variety of components of interest to users of the Data Items. It has also been prompted by experience in analysing engineering structural failures where it is apparent that these have been triggered by very sharp stress concentrations that lie in areas which may be treated alternatively through finite elastic stress concentration theory and fracture mechanics.

The new features in this paper are principally the approach to treat interacting geometric stress concentrations and external steps or 90 degree corners. The increasing use of finite element stress analysis is also addressed in the sense that the strategies described in the paper are shown as complementary to FEA.

\section{Tom Gray}

Emeritus Research Professor

Department of Mechanical Engineering

University of Strathclyde

Glasgow G1 1XJ 


\title{
Fracture Mechanics approach to design analysis \\ of notches, steps and internal cut-outs in planar components
}

\author{
T. G. F. GRAY, J. WOOD \\ Department of Mechanical Engineering \\ University of Strathclyde \\ Glasgow G1 1XJ
}

\begin{abstract}
A new approach to the assessment and optimisation of geometric stress-concentrating features is proposed, based on the correspondence between sharp crack or corner stress-field-intensity factors and conventional elastic stress concentration factors for radiused transitions. This approach complements the application of finite element analysis and the use of standard SCF data from the literature. The method makes it possible to develop closed form solutions for stress concentration factors, in cases where corresponding solutions for the sharp crack geometries exist. This is helpful in the context of design optimisation. The analytical basis of the correspondence is shown, together with the limits on applicability where stress-free boundaries nearby the stress concentrating feature are present or adjacent features interact. Examples are given which compare parametric results derived from finite element analysis with closed form solutions based on the proposed method. New information is given on the stress state at a $90^{\circ}$ corner or width step, where the magnitude of the stress-field-intensity is related to that of the corresponding crack geometry. This correspondence enables the user to further extend the ap-
\end{abstract}


plication of crack-tip stress-field-intensity information to square-cornered steps, external Ugrooves and internal cut-outs.

Key Words: stress concentration factor, notch interaction, notch shielding, slots, steps, Ugrooves.

\section{Introduction}

Stress concentration effects at notches, steps and cut-outs in engineering components are responsible for a high proportion of fatigue failures. However, such features cannot always be avoided and the best that can be done normally is to reduce the stress concentration effects by optimising the geometry of the offending details. At present, there are two basic approaches to initial design, assessment or improvement of stress concentrating details. The first is to make use of published stress concentration factors (SCFs) for standard notch shapes for example, as compiled by the Engineering Sciences Data Unit [1]. The second option is to make use of finiteelement analysis (FEA) facilities, which are now widely available to practising engineers. In the latter case, it is also possible to apply optimisation software, which will alter the geometry of the detail in prescribed ways and thereby arrive at an improved design solution.

There are two common practical weaknesses in the use of published stress concentration factors - apart from the usual problem of finding cases in the literature that match the design situation under consideration. The first is that much of the data are based on photoelastic determinations and do not as a consequence cover very sharp concentrations, i.e. with small radii. Various references to photoelastic determinations, going back to Frocht's early work [2], note that experimental errors may be large if the notch radii are very small in comparison with the specimen thickness. The second difficulty arises typically where the component in question fea- 
tures several contiguous stress concentrating details that interact, sometimes in a manner which reduces the SCF and sometimes not.

The finite-element approach is more versatile in treating stress concentration problems, but gives less insight, for example, in terms of distinguishing the interacting effects of notch position and transition radius. Local mesh refinement will be required at each detail on a component being analysed, leading to extensive computational requirements, particularly in a 3D analysis, and multiple runs in a parametric design study (although this is becoming a less significant problem as computer power increases). In the case of small radii, it may not always be clear that the mesh has been refined enough and this may require further exploration or sub-structuring of the model. The finite element results presented in this paper were produced using an alternative approach based on so-called "adaptive p-element" technology, where the polynomial shape function along each edge of the elements is varied until a specified convergence is achieved (detailed aspects of this application are given in the Appendix). FEA of one kind or another can therefore provide accurate stress concentration factors for non-standard geometries and loadings. However, single determinations of SCF do not readily inform the designer how to optimise the geometry of the detail.

A third approach proposed here, therefore, is to analyse stress concentrating features through separate modelling idealisations. In the first step, all radiused or smoothed transition features are treated as sharp cracks or square corners with zero radius. They can then be analysed and characterised in terms of crack-tip stress-field intensity factors (SIFs) or equivalent parameters. The SIF can be determined analytically, by drawing on a large database of published crack stress-intensity factors, together with the superposition and 'compounding' methods available, as given by Murakami [3] and Rooke et al [4]. Alternatively, the SIF may be determined numerically through FEA, in which case the modelling requirement is much reduced relative to the 
radiused notch model. The true transition shape does not need to be described accurately and special crack-tip singularity elements or the approach described in the appendix may be used to deal with the problem of mesh refinement.

Having established the basic influence of the size and spacing of the cracks or corners in terms of stress intensity factors, the effect of transition shape (eg corner radius) can then be found through analytical functions linking crack stress-intensity factors and stress concentration factors for the corresponding rounded notches. The analytical underpinning inherent in this approach is also beneficial in terms of providing a parallel form of analysis, which serves to validate the computational result. Detailed finite-element analysis may then be applied to the chosen geometry as a final check on the stress concentration factor.

\section{Analytical correspondence between SIF and SCF (isolated SCs)}

In the case of symmetrical internal or external notches, the analytical correspondence between sharp cracks and rounded notches has been expressed [5] in terms of a generalisation of the simple formula for the SCF at an elliptical hole, subject to a uniaxial stress $\sigma_{\infty}$ normal to the appropriate line of symmetry. The stress concentration factor, $K_{\text {scf }}$, is given by:

$$
K_{\mathrm{scf}}=\frac{\sigma_{\max }}{\sigma_{\infty}}=1+2 Y \sqrt{\frac{a}{r_{o}}}
$$

where $Y$ is the well-known crack configuration factor, $a$ is the crack/notch depth and $r_{o}$ is the instantaneous radius of the notch at the end of the line of symmetry.

This generalisation of the elliptical hole solution is supported by the 'blunt crack' theory of Creager [6] which was formulated in terms of the sharp crack SIFs associated with the three Irwin 
loading Modes. Hyde and Yaghi [7] have also used Creager's equations to determine SCFs for narrow rectangular notches with semi-circular ends. Dini and Hills [8] considered the correspondence between the stress fields at notches and corresponding crack cases, in order to determine the extent to which the radius perturbs the singular stress field of the crack solution. Tada et al also noted that in the case of the infinite plate, (i.e. where $Y=1$ ) equation (1) is "actually not limited to slender ellipses" [9].

However, equation (1) can carry the crack/notch analogy further than previous applications, by allowing separation of the configuration factor $Y$ from the other terms in the stress intensity factor formulation. The claim inherent in equation (1) is that the $Y$ factor can be used to generalise the elliptical-hole SCF expression in terms of different boundary configurations and loadings. The further inference is that equation (1) can also correctly describe large-radius, wide notches of depth $a$. In other words, the elliptical hole solution for the 'shallow' side of the hole can also be employed. This formulation, confirming Tada's view stated earlier, is not therefore confined to narrow 'crack-like' notches, where the correspondence between the crack and notch solutions would not be remarkable.

A variation of this principle in terms of the Neuber hyperbolic [10] notch solution was also suggested in reference [5], to cover the case of deep, opposed notches of relatively large radius, where the 'neck' between notches is small. In that case, the corresponding SCF for tension loading (again, in terms of the gross stress $\sigma_{\infty}$ ) is given by:

$$
K_{\mathrm{scf}}=\frac{2\left(d / r_{o}+1\right) \sqrt{\left(d / r_{o}\right)}}{\left(d / r_{o}+1\right) \operatorname{arctn}\left[\sqrt{\left(d / r_{o}\right)}\right]} \frac{1}{Y_{\mathrm{hyp}}}
$$


where $d$ is the net section width between the notches and $Y_{\text {hyp }}$ is an equivalent configuration factor for the hyperbolic solution. This is determined by equating the theoretical stress concentration factors formulated in terms of the elliptical hole and hyperbolic notch solutions.

The reasons for this simple correspondence between notch and crack solutions in finite geometries are discussed in greater detail in reference [5]. However the basic point is that, through a consequence of Saint Venant's Principle (and subject to certain restrictions) the configuration factor for a crack geometry in a given case is likely to be very similar numerically to the equivalent configuration factor for an analogous notch. Detailed examination in reference [5] of finiteelement results for a wide variety of large and small-radius, shallow and deep notches confirmed the applicability and scope of equations (1) and (2). The study included tension and bending cases with SCFs varying from 1.2 to 13 (net stress basis).

Equation (1) also applies in the case of notches which are asymmetric with respect to the loading axis, as exemplified by an external step, although the stress intensity factor concept then needs to be interpreted differently. Asymmetry leads to mixed-Mode stress distribution around the sharp corner, as shown in the left-hand detail in figure 1 and requires a different basis for characterisation, distinct from a single-Mode stress-intensity factor. The problem can be treated in terms of a 'sharp-corner' stress-intensity factor [11] derived from the sharp-corner 'energy release rate', calculated numerically as a variation of energy with step depth. (This parameter has no physical significance in fracture terms, it merely provides a characterising framework for the corner stress distribution and facilitates extension to the stress concentration factor at a rounded corner.)

Comparing symmetric and asymmetric notches with the same depth and transition radius, as in figure 1 , the main effect of asymmetry is to reduce the SCF considerably relative to the corre- 
sponding symmetric case. An empirical adjustment to equation (1) to achieve this is given in reference [11] and the sharp corner problem will be discussed further in section 4 .

\section{Interaction of stress concentrations}

\subsection{Comparison of stress distributions (notches $v$ cracks)}

Further questions concerning the correspondence of crack and notch solutions arise when the notches are not 'isolated', but are placed near to other notches or to free boundaries. The principal differences between crack stress distributions and corresponding notch patterns lie in the extent of the region that experiences a raised stress level. Figure 2 has been constructed using the Inglis complex variable equations for the stresses round an elliptical hole subject to uniaxial load [12]. Three cases are considered - a circular hole, a 2/1 elliptical hole and a crack (actually a 1000/1 ellipse).

Figures 2 (a) and (b) show that, in all three hole geometries, the stresses normal and transverse to the major axis of the ellipse reduce to almost identical levels by two to three half-cracklengths from the notch end. When the applied load is parallel to the major axis, the transverse stresses converge even more rapidly (figure 2 (c)). However the convergence of stress levels is more gradual for stresses in the same direction as the load, requiring an overall distance nearer six half-crack lengths - as in figure 2 (d).

Several useful deductions can be made from this comparison. Firstly, the effect on SCF of placing a free edge parallel to the loading direction should be very similar for cracks and notches, provided that this boundary is at least three half-crack-lengths from the end of the notch. However, this statement needs to be qualified in at least two ways:

i. The argument is qualified for holes or notches that have a larger length-to-width ratio than the circular case, although such notches have small SCFs in any event; 
ii. Following St Venant's Principle, a small discrepancy between the crack and hole solutions, with respect to the differences in stress at a boundary, may nevertheless have a strong influence if the error extends over a large length in comparison to the distance to the point where the SCF is to be determined. The famous error in Griffith's 1921 paper on energy release rate [13] provides a classic example of this. He used Inglis' elastic solutions to calculate the boundary work at the perimeter of a large plate containing a crack-like elliptical hole and compared this to the energy in an uncracked plate with uniform boundary stress. The Inglis stress values differ from the assumed uniform stress only by an infinitesimal amount and are statically equivalent, but the fact that the error occurs over a perimeter length which is always greater than the distance to the crack means that the error can never be negligible.

In contrast, if the free boundary is inserted at a distance less than about one hole radius from the notch end, especially in the case of a large-radius notch, the region at elevated stress is much greater than it would be for a crack. The stress concentration at such a notch will therefore be substantially increased (relative to the crack analogue solution embodied in equation (1)). Likewise, following figure 1(d), the stress concentration effect will be increased if the boundary which is loaded is placed nearer than six hole radii from the edge of the hole.

Figure 3(a) shows SCF results obtained via finite-element analysis for a single hole placed centrally in a plate of width $2 \mathrm{~W}$. These results cross-reference accurately with an empirical, closedform equation given in Roark's Formulas for Stress and Strain [14] which has its origin in photoelastic tests (CF Roark, ie Closed Form Roark). The finite-element values are then compared with closed form SCFs (CF centre hole) derived through equation (1). The crack configuration factor used here was based on a simple closed formulation for a centre-crack geometry [15] 
(equation 3) which also agrees to within $1 \%$ with the most accurate of several closed forms given for the centre-crack geometry in reference [9].

$Y=1 /\left(1-\left(\frac{a}{W}\right)^{1.84}\right)^{0.54}$

The centre-crack closed form clearly underestimates SCFs for holes larger than about $30 \%$ of the finite width - in line with the suggestion earlier in this section on the effect of proximity to a free boundary. In the case of very large holes, the error is substantial, as the stress pattern in the narrow ligament is then dominated by additional bending effects not present in a cracked geometry. This circumstance violates condition (ii) above. Fortunately, such interactions are more often associated with situations of lesser practical significance, where the SCF values are small on a net stress basis (less than 2.0). This may arise where the transition radius is large and the notch is deep or wide relative to the total cross section. Such cases can sometimes be treated by substituting the hyperbolic notch analysis for the elliptical hole solution, as indicated earlier. This substitution also deals with the limiting case when $r_{o}$ tends to infinity and the SCF must reduce to unity on a net stress basis (equation (1) does not show the correct limiting trend in such an extreme case).

Figure 3(b) shows finite-element results for an infinite row of holes of radius $a$ spaced $2 W$ apart transverse to the load ('FE periodic') and compares these with a closed form estimate based on equation (1) and the classical solution for a transverse row of cracks ('CF periodic'). It is of interest to note in passing that, although the crack configuration factor for this case is non-linear with respect to $a / W$, the overall dependence of the net-stress-based SCF on width-ratio is linear. The periodic crack solution is remarkably effective for holes that are large compared to the spacing. This is because the adjacent boundaries in this case are other holes and not straight edges, and they are reasonably far apart. A similar point was made by Rooke et al [4] in the 
context of 'compounding' and the effects of different types of adjacent boundaries on the stress intensity at a crack.

\subsection{Stress Multiplication and Notch Shielding}

A 'stress flow' analogy is often invoked to provide a measure of intuitive understanding of stress concentration effects and two extremes can be recognised in the case of multiple notches. If the notches or cracks are placed in line across the load path, the stress concentration effect is clearly more severe than for a single notch in an infinite width (as in the example of periodic circular holes given earlier). Also, a small-scale stress concentration, such as a small hole, situated in the highly stressed zone adjacent to a larger-scale hole may be treated conservatively by multiplying the respective individual SCFs. A more accurate approach might make use of the configuration factor for a crack adjacent to a hole, through equation (1), and thereby take reasonable account of the stress gradient adjacent to the large hole.

If, on the other hand, the notches are placed along the load path, one behind the other, the stress concentration effect is reduced somewhat for the end notches in the series and even more for the intermediate notches, where a measure of 'shielding' takes place. These effects can be estimated from appropriate crack solutions, subject to the limitations given in 3.1 when the zones of elevated stress begin to encroach on each other or on free boundaries.

To explore this possibility, an extended solution was generated, via FEA, for three cracks in series along the load path, as shown in Figure 4 (again, see Appendix for details). These results were confirmed for $a / h \leq 0.8$ by data in reference [3], where a closed form fit is given for the outer cracks as:

$Y(a / h)=1-0.0031(a / h)-0.5036(a / h)^{2}+0.3424(a / h)^{3}$ 
and tabulated values are given for the inner cracks. (The FEA values were actually generated for a finite-width plate ( $a / W=0.1$ ) and adjusted to compare with the infinitely-wide case in reference [3] by a method which will be described later). As the cracks are brought closer together, the configuration factors appear to reach limiting values of 0.53 and 0.73 approximately for the inner and outer cracks respectively.

Figure 5 gives corresponding FEA results for stress concentrations at the inner and outer of a group of three circular holes placed in line in a relatively wide plate $(a / W=0.1)$ (note false zero on SCF scale). The results are compared with the values ('CF a,b') derived from equation (1), where the main influence arises from the crack configuration factors given in figure 4 for the inner and outer cracks. Equation (4) can be applied to this case, as it is valid down to a spacing corresponding to $a / h \leq 0.8$ and circular holes will coalesce at $a / h=1$.

The effect of the finite width, although small, was included in the determination of the closed form estimate in figure 5 by multiplying the result from equation (4) by the adjustment indicated in equation (3) for a single crack in a finite width. The justification for multiplying these factors lies in an explanation based on Cartwright and Rooke's compounding method [16],[17]. If the separate configuration factors for a crack near to different boundaries are given by $(1+\alpha)$ and $(1+\beta)$, the first stage of Cartwright and Rooke's method, based on an alternating boundary stress technique, gives the compounded factor as $\left(1+\alpha+\beta+k_{2}\right)$, where $k_{2}$ is a factor related to mutual interaction of the two disturbing boundaries. This should be negligibly small unless these boundaries are near each other. Multiplication of configuration factors, on the other hand, gives $Y=(1+\alpha+\beta+\alpha \beta)$ and, if $\alpha$ and $\beta$ are small $\alpha \beta$ will be an order smaller, thus approximating to the compounding approach. This method was also applied in figure 4, where the FEA results were divided by the result from equation (3) to generate a comparison with the infinitely-wide geometry of reference [3]. 
In the case shown in figure 5 , the SIF-based solution should only be accurate if the ligament between the holes is more than about six times the hole radius. However the crack-based approximation now under-estimates the 'sheltering' effect (ie over-estimates the SCF) for more closely spaced holes. This is in line with the discussion in 3.1 concerning the stress gradient along the axis parallel to the load. It is also seen that the error is greater for the inner hole, which has a disturbing boundary on both sides, than for the end holes. Nevertheless, bearing in mind the magnified ordinate scale in this diagram, the estimate of SCF is still remarkably good and entirely adequate for design purposes.

Figure 6 shows a similar comparison between FEA results for parallel-sided slots with semicircular ends ('FE a,b') and the corresponding SCF values generated through equation (1) ('CF $\left.a, b^{\prime}\right)$. The configuration factors were derived from the previous finite-element analysis of the corresponding three-crack geometry shown in figure 4. Relative to the previous circular hole case, the slot pitch could in practice be reduced substantially without overlap and the full range of the results in figure 4 is therefore needed. The finite-width ratio $(a / W)$ was again 0.1 and the slot radius was $40 \%$ of the half-crack-length. Comparison with figure 5 shows that the closed form is more accurate than is the case for the circular holes. This arises because the stress pattern in the 'sheltered' area between the slots is closer to the crack case than the case for circular holes. The finite-element results also show that, for a given spacing, the 'sheltering' effect is greater in the case of sharp slots than for circular holes.

\section{Square-cornered steps, U-grooves and cut-outs}


Mode I stress intensity factors at square corners may be determined in an approximate manner by first considering the decomposition of separate force systems applied to a classical crack in an infinite plate, as shown in figure 7(a). For load case $P$, superposition of the two loading systems $Q$ and $R$ gives: $K_{\mathrm{I} P}=K_{\mathrm{I} Q}+K_{\mathrm{I} R}=2 \sigma \sqrt{\pi a}-\sigma \sqrt{\pi a}$. As the $Q$ and $R$ loading systems are identical, $K_{\mathrm{I} Q}=K_{\mathrm{I} R}$, and therefore $K_{\mathrm{I} Q}=0.5 \sigma \sqrt{\pi a}$. (Note that this is close to the limiting configuration factor for the inner crack in the 3-crack case shown in figure 4.)

An infinitely-long cut-out or external step can then be generated by creating an internal, stressfree block as shown in figure $7(\mathrm{~b})$ to give a formulation for stress concentration as:

$$
K_{\text {scf-cut-out }}=1+2 \times(1-0.5) \sqrt{\frac{a}{r_{o}}}
$$

(A similar idea was suggested by Noda et al in the context of stress concentration factors for shoulder fillets [18].) However, by analogy with the well-known edge-crack stress intensity adjustment, a slight increase in stress intensity factor is implied, due to release of transverse stresses in the released block. Comparison of SCFs for transverse, internal, round-end slots and comparable long rectangular cut-outs suggests that this increase varies little from $10 \%$ (cf classical edge factor increase of $12 \%$ for a semi-inifinite crack). Hence, a combined $Y$ configuration factor for a long, sharp-cornered, internal cut-out or hole in an infinitely wide plate might reasonably be taken as 0.55 , ie $1.1 \times(1-0.5)$.

If the infinite geometry in figure $7(b)$ is then split to form two semi-infinite plates with symmetrical external steps, as in figure $7(\mathrm{c})$, there ought to be a further, rather smaller, release of transverse stresses at the cut edge. Noda's numerical results [18] for an infinitely long external step, based on a body-force analysis method, gives a combined edge factor that varies only a little from a value of 1.16 , over a very wide range of $a / r_{o}$. This implies that the split to form the semi-infinite 
plates generates a further $5 \%$ increase in stress intensity factor and therefore gives the Mode 1 $Y$-factor for a sharp cornered external step in a semi-infinite geometry as 0.58 . Hence the stress concentration factor for the case in $7(\mathrm{c})$ is given by:

$$
K_{\text {scf-ext step }}=1+2 \times 1.16 \times(1-0.5) \sqrt{\frac{a}{r_{o}}}
$$

The accuracy of this equation ('External step CF') relative to Noda's results, is shown in figure 8, which covers a wide range of external steps, from vanishingly shallow to relatively sharp.

General comparison with other published SCF data is complicated by the mixed-Mode nature of the stress distribution and the fact that the maximum stress location at a corner does not coincide with the tangent between the radius and the straight boundary, as shown in figure 1 . The above result is of the same order as the mixed-Mode factor of 0.66 found rather less accurately in [5] by extrapolation of finite-element results to zero radius, but it is of interest to investigate this point more closely through FEA.

Figure 9 shows the corresponding crack and corner stress-field-intensity magnitudes in a polar coordinate system centred on the crack tip or analogous sharp corner, at a point very close to the origin where singular stress patterns dominate. This formulation is based on the circumferential stress and the singular regions were identified by plotting stress versus radius on logarithmic scales. The maximum stress at the corner occurs at an angle of $-30^{\circ}$ (see figure 1 ) and has a magnitude of $63 \%$ of that generated by the corresponding crack geometry. The strength of the singularity varies with angle in the corner case, but at the angle giving maximum stress, it shares, with the crack geometry, the same inverse square root dependence on radius. From all these considerations, it is reasonable to estimate the reduction effect of asymmetry in the twodimensional corner case as $60 \%$ approximately. 
In the case of internal cut-outs or external steps of finite length (see right-hand sketch in figure 10) an estimate of the crack face stress to be released, as in figure $8(b)$, can be made from solutions [3] [9] for a pair of cracks spaced at a distance $2 h$ along the load axis. These independent solutions, referenced by Murakami and Tada respectively, agree reasonably in the case of the Mode 1 factor for $h / a>0.1$. However, they diverge for more closely spaced cracks. A finiteelement cross-check suggests that mixed-Mode effects dominate in the range where the parallel ligament between the cracks tends to vanishing thickness. Hence, as a limit of $h / a>0.1$ is probably well within the range of practical interest, it will be accepted here.

These comparisons suggest that the closed form given by Murakami gives a good estimate of effective configuration factor viz;

$Y_{h / a}=1-0.293 s\left(1-(1-s)^{4}\right)$

where $s=1 /\left(1+\frac{h}{a}\right)$

Putting this together with the superposition algorithm above and equation (1) leads to closed form stress concentration factors as follows:

$$
\begin{aligned}
& K_{\text {scf-cutout }}=1+2 \times 1.1 \times 1.01 \times\left(1-0.5 Y_{h / a}\right) \sqrt{\frac{a}{r_{o}}} \ldots \ldots . . . \\
& K_{\text {scf-Ugroove }}=1+2 \times 1.16 \times 1.01 \times\left(1-0.5 Y_{h / a}\right) \sqrt{\frac{a}{r_{o}}} . . .
\end{aligned}
$$

for finite length internal rectangular cut-outs and external U-shaped notches respectively. (The factor 1.01 has been included to allow comparison with the finite element determination for a finite-width corresponding to $a / W=0.1$.) 
Comparison is shown in figure 11 of closed form equation 8(a) (Cutout CF) with finite-element results for rectangular holes or cut-outs with radiused corners, in a finite-width plate $\left(a / r_{o}=2.5 ; a / W=0.1\right)$. Equation (8a) underestimates the SCF as the axial length of the cutout is reduced $(h / a \rightarrow 0)$ as expected. At the point $h / a=0.2$, the two notional parallel slits making up the cut-out merge into a single slot. Therefore, for narrower spacing, down to the point $h / a=0$, it is more reasonable to model the geometry as a single slit with semicircular ends, as shown by the legend 'Radius CF'.

In the case of external U-grooves, equation (8b), as given, was found to give a slightly low (-3\%) estimate of SCF in the limiting case of an infinitely-wide, external U-groove, where the finiteelement determination does not seem to converge to Noda's results. An edge configuration factor of 1.2 in equation (8b) has therefore been used in figure 12 (instead of 1.16) to provide improved agreement with the finite-element data, but the differences are small and may simply be related to the definition of maximum stress locations.

\section{Summary}

The scope of a previously given analytical relationship between crack-tip stress intensity factors and stress concentration factors has been explored further for various two-dimensional geometries subject to symmetrical loading.

Several configurations, incorporating circular holes and parallel-sided slots with semi-circular ends, have been examined. The large radius relative to the 'crack length' in the cases incorporating circular holes cases implies a significant departure from a crack-like shape discontinuity. Despite this, the analytical relationship, based on crack configuration factors, gives usefully ac- 
curate estimates of stress concentration factors - provided that given minimum distances are respected between the circular holes and adjacent free boundaries. In the case of stress concentrating details that have a smaller aspect ratio (dimension transverse to the load relative to end radius) it is deduced that the analytical equation will be effective at closer spacing. The required minimum spacing for a given aspect ratio may be estimated from Inglis' equations, used to draw up a stress plot for an appropriately dimensioned elliptical hole.

Stress concentrations at corner details have also been examined to explore the effects of longitudinal spacing between adjacent corners. A new analytical formulation has been given for $90^{\circ}$ radiused corners joined by a flat or parallel section. This is based on the published configuration factor for a pair of transverse cracks, placed in series along the load axis. Provided that minimum spacing is again respected between the adjacent faces of the cut-out or external notch, the formulation should be useful in the design context.

The results of the study provide support and background information for the proposed design strategy whereby stress concentrating details may be treated initially as mathematically sharp transitions (cracks or corners) for the purpose of FEA. The analytical approach also provides an appropriate design framework where FEA is not available or where an independent crosscheck on the result of a finite-element analysis is required.

\section{Conclusions}

Comparison of FEA-derived stress concentration data with corresponding estimates derived through configuration factors for analogous crack geometries confirms the usefulness of a previously given generic equation. The required configuration factors may be obtained from the published literature or generated through FEA analysis of the appropriate cracked body. 
The accuracy of the estimate for multiple notches, or notches which approach free boundaries, may be gauged by considering the differences between the analogous crack/notch stress fields and ensuring that certain minimum distances between adjacent notches or boundaries are respected.

In the case of adjacent similar notches, the generic equation tends to underestimate the SCF for notches placed across the predominant loading axis, whereas the method tends to overestimate the SCF (and underestimates the 'sheltering effect') for notches positioned along the load axis.

An extension of the method has been given for finite-length, rectangular holes with rounded corners or corresponding external U-grooves, where the design aim may be to find an acceptable compromise between the corner radius and spacing of shoulders.

\section{Appendix}

\subsection{Nomenclature}

a crack length

$d \quad$ width of 'neck' between deep notches

$h \quad$ axial spacing of cracks or stress concentrations

$r \quad$ radius coordinate from crack tip or corner

$r_{o} \quad$ asymptotic radius of notch end

$s \quad$ geometric parameter (see equation 7)

$K_{\mathrm{I}} \quad$ crack-tip stress-field-intensity factor 
$K_{\text {scf }}$ stress concentration factor

$W \quad$ half width of finite-width geometry

Y configuration factor

$\theta \quad$ polar coordinate

$\sigma_{\infty}$ remotely applied stress

$\sigma_{\max }$ maximum stress at a stress concentration

\subsection{Application of Adaptive P-element technology}

The finite element results in this paper were produced using two-dimensional plane strain elements, as implemented in the Pro-Mechanica Applied Structure system from Parametric Technology Inc. Adaptive p-element technology allows the user to specify a percentage convergence for the analysis and, in the analyses reported here, convergence was based upon displacements, strain energy and a global root-mean-square stress measure. Convergence levels achieved were typically much better than $1 \%$.

For the analyses of cracked configurations, linear elastic stress intensity factors were derived by fitting stress distributions calculated in the vicinity of the crack tip to the Mode 1 Westergaard stress equations. The stress fitting was performed along a user-specified line segment, as shown in figure 13. It should be noted that the polynomial shape function along each edge of the p-elements can be as high as 9th order. Mechanica uses a 3-term fit for the Westergaard 
equations, which is valid for moderate distances from the crack tip, while smoothing out any "noise" in the stress data.

For all analyses, the fan of elements around the crack tip was set to a radius of $1 / 20$ th crack length and the next larger fan was set to $1 / 4$ crack length. The latter radius normally defined the outer limit for the crack tip singular stress fit.

Another particularly useful feature of the Mechanica system is the facility to set up geometrical parameters (e.g. distance between multiple cracks) as variables and to automatically carry out sensitivity studies, by varying the parameters between user-specified limits. Where this facility was used, the adaptive-p mesh refinement process was repeated for each increment of the geometrical parameter, until the required convergence was reached.

\section{References}

1. Elastic Stress Concentration Factors - geometric discontinuities in flat bars or strips of isotropic material, Data Item 69020 with amendments A, B C, Engineering Sciences Data Unit, London, 1993.

2. Frocht, M.M, A photoelastic investigation of stress concentrations due to small fillets and grooves in tension, Technical Notes, National Advisory Committee on Aeronautics, Washington, 1951, 2442.

3. Murakami, Y., ed., Stress Intensity Factors Handbook, 1987, Pergamon Press, Oxford.

4. Rooke, D., Baratta, F.L. and Cartwright, D.J., Simple methods of determining stress intensity factors, Eng Fracture Mech., 1981, 14, 397-426.

5. Gray, T. G. F., Tournery, F., Spence, J., Brennan, D., Closed form functions for elastic stress concentration factors in notched bars, J Strain Analysis, 1995, 30, 2, 143-154. 
6. Creager, M., The elastic stress field near the tip of a blunt crack, MSc thesis, 1966, Lehigh University.

7. Hyde, T.H. and Yaghi, A., Peak stresses near narrow rectangular notches, with rounded corners, subjected to tensile and shear loading, J Strain Analysis, 1993, 28, 1, 5-11.

8. Dini, D., and Hills, D.A., The effect of a crack-tip radius on the validity of the singular solution, $J$ Mech Eng Sci, 2004, 218, 7, 693-701.

9. Tada, H., Paris, P.C., Irwin, G.R., The Stress Analysis of Cracks Handbook, 2000, ASME.

10. Neuber, H., Theory of Notch Stresses, 1958, Springer Verlag, Berlin.

11. Gray, T.G.F., Tournery, F., Spence, J., Analysis of stress concentration factors for stepped plates based on a crack tip stress intensity approach, J Strain Analysis, 1995,30, 2, 143-154.

12. Timoshenko, S., and Goodier, J.N., Theory of Elasticity, 2nd Ed., 1951, McGraw-Hill, New York.

13. Griffith, A.A., Stress Concentrations in Theory and Practice, 1921, British Association Report, p316.

14. Young W. C., Roark's Formulas for Stress and Strain, $6^{\text {th }}$ Edition, 1989 , McGraw Hill, New York.

15. Gray, T.G.F., Convenient closed form Stress Intensity Factors for common crack configurations', I J Fracture, 1977, 13, pp 65-74.

16. Cartwright, D. J., and Rooke, D. P., Approximate stress intensity factors compounded from known solutions, Eng Fract Mechs, 1974, 6, 563-571.

17. Gray, T. G. F., Applicable Fracture Mechanics', PhD thesis, 1978, University of Strathclyde.

18. Noda, N-A., Takase, Y. and Monda, K., Stress Concentration Factors for shoulder fillets in round and flat bars under various loads, Int J Fatigue, 1997, 19, 1, 75-84. 


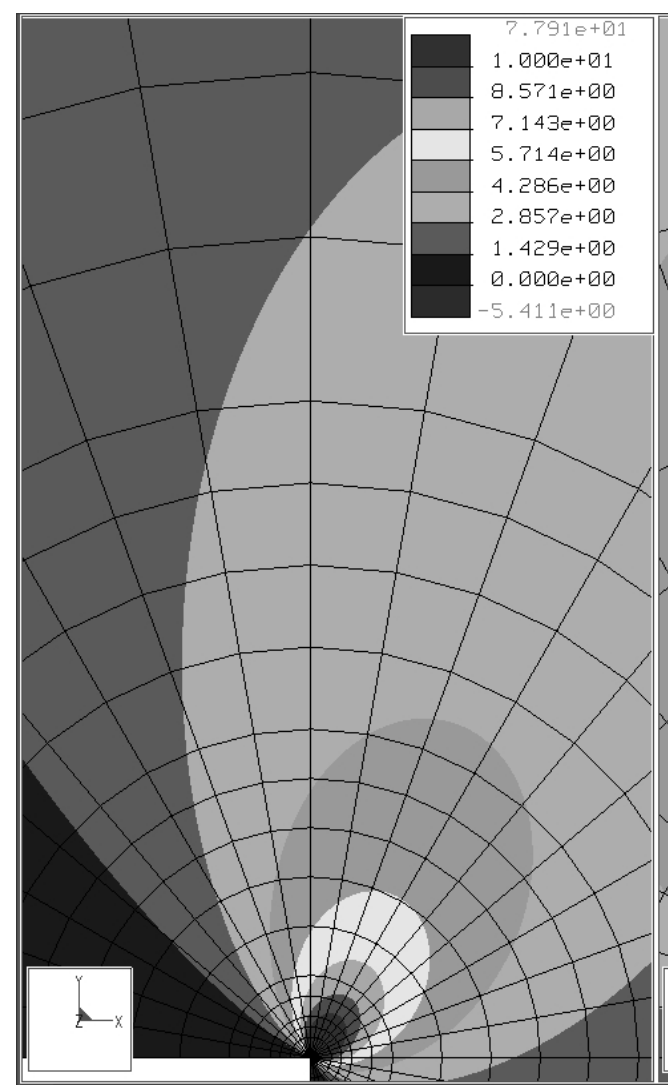

Corner

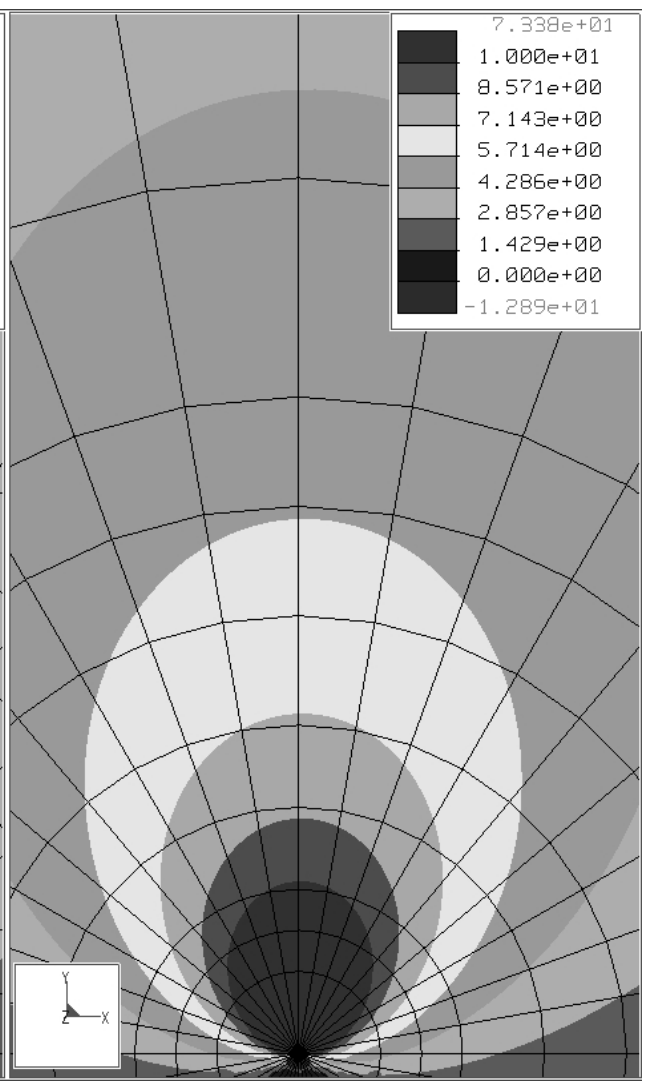

Crack

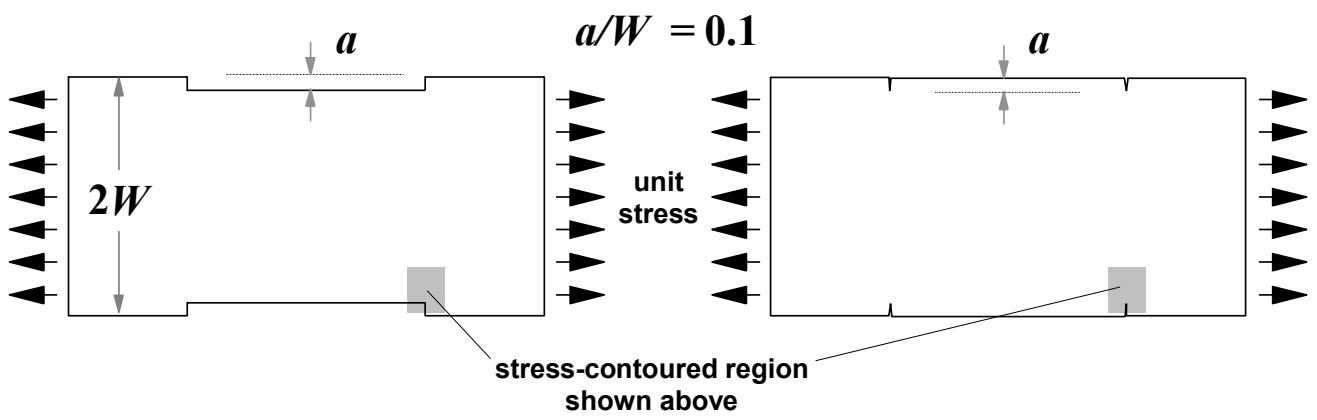

Comparison of circumferential stress contours between equivalent cracks and corners

Figure 1 


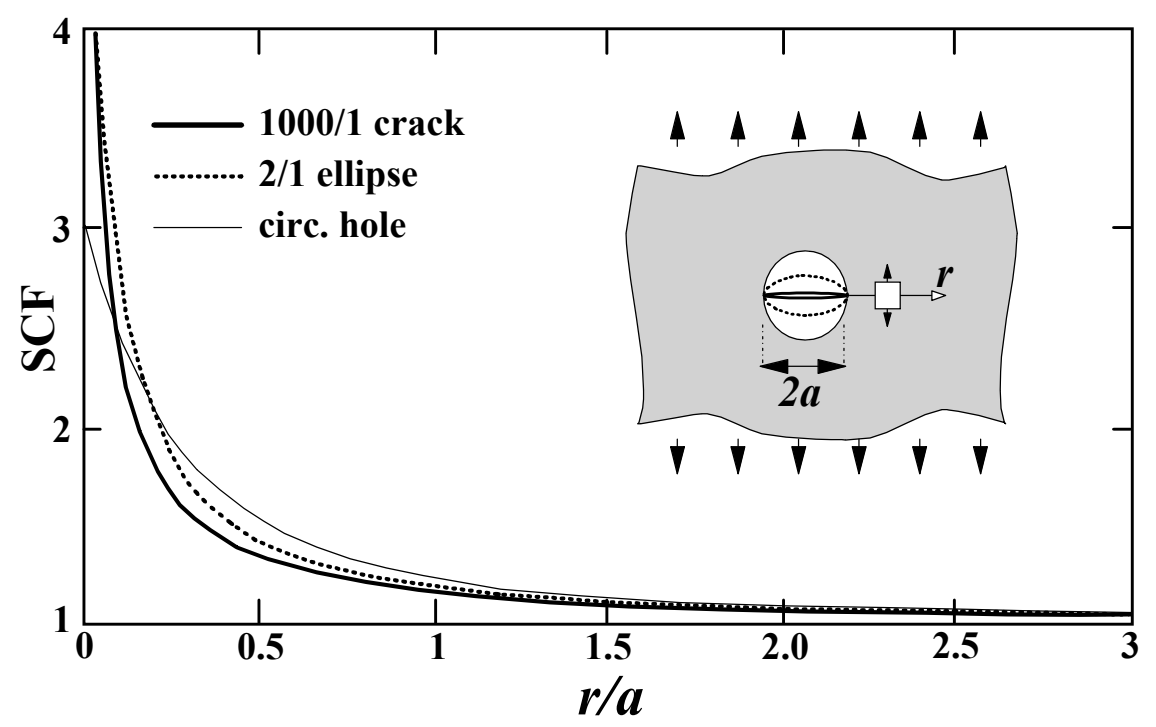

Notch/crack stress parallel to load on plane of major axis

Figure 2(a)

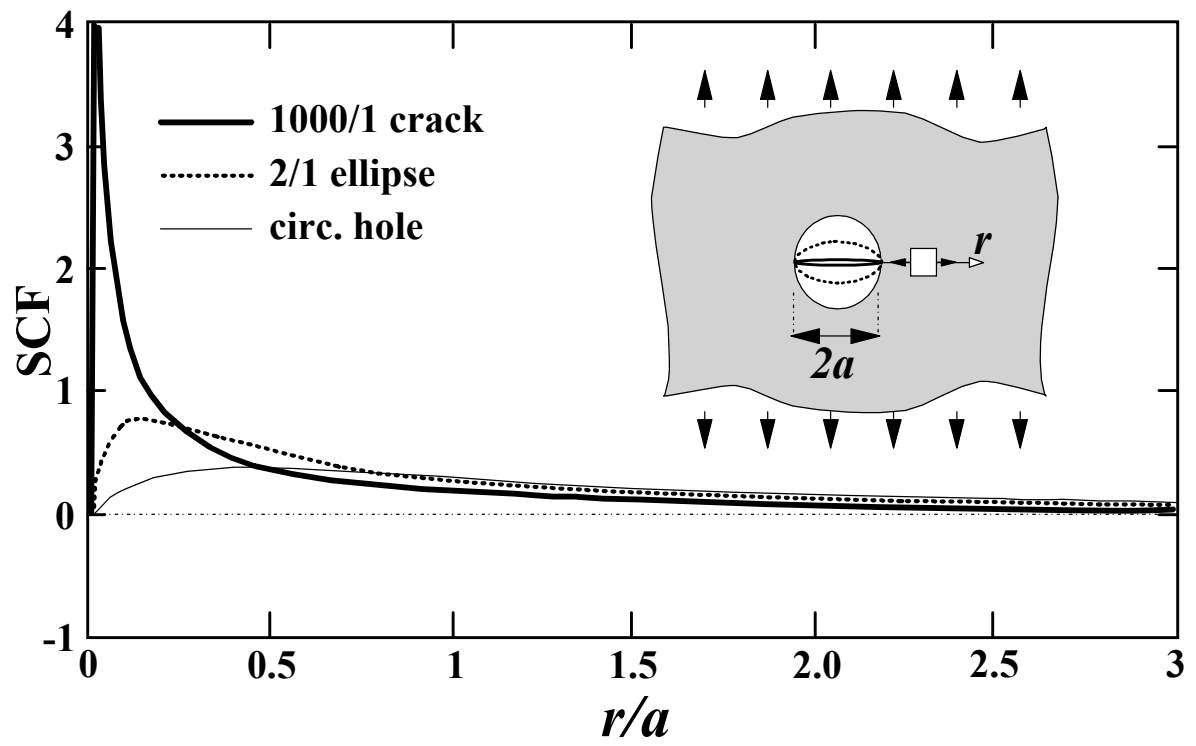

Notch/crack stress transverse to load on plane of major axis

Figure 2(b) 


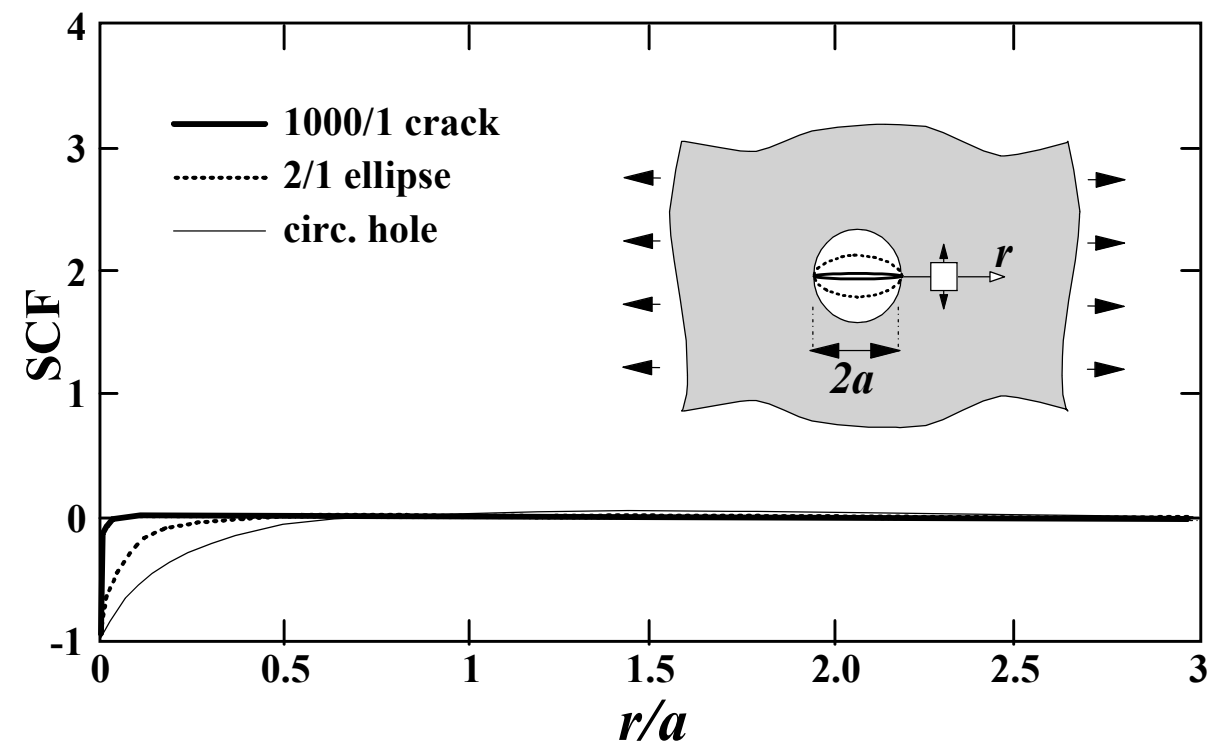

Notch/crack stress transverse to load (major axis aligned with load)

Figure 2(c)

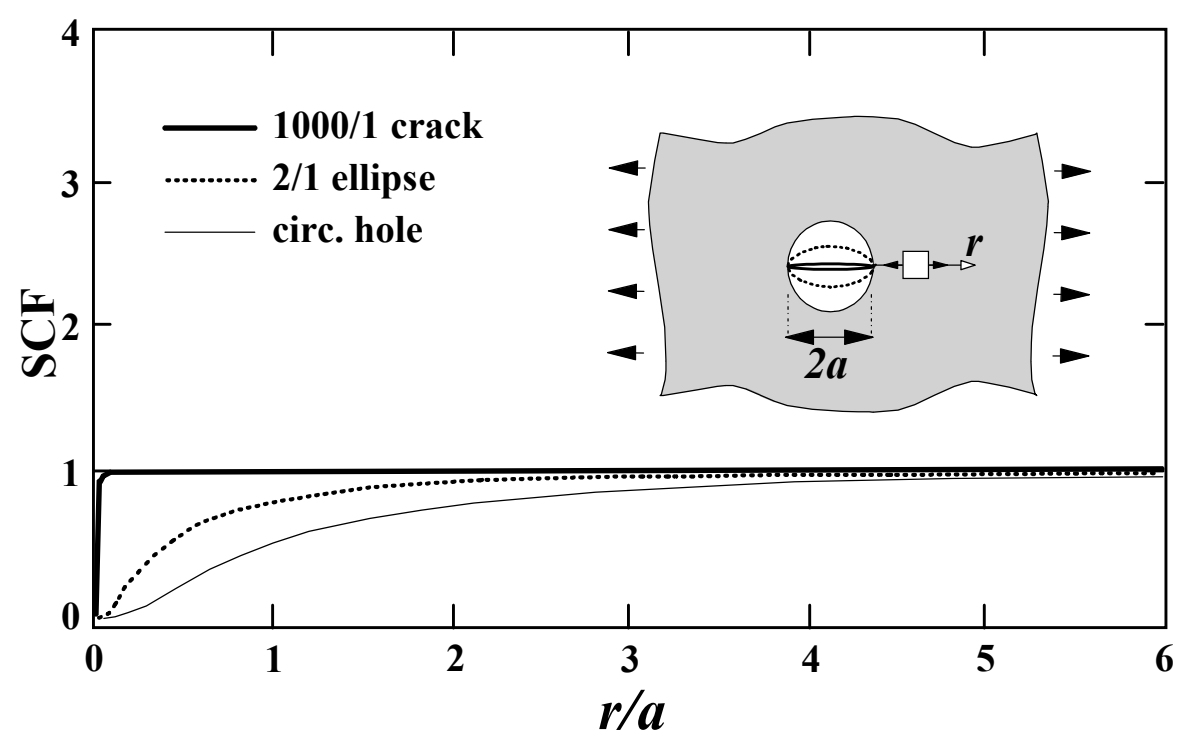

Notch/crack stress parallel to load (major axis aligned with load)

Figure 2(d) 


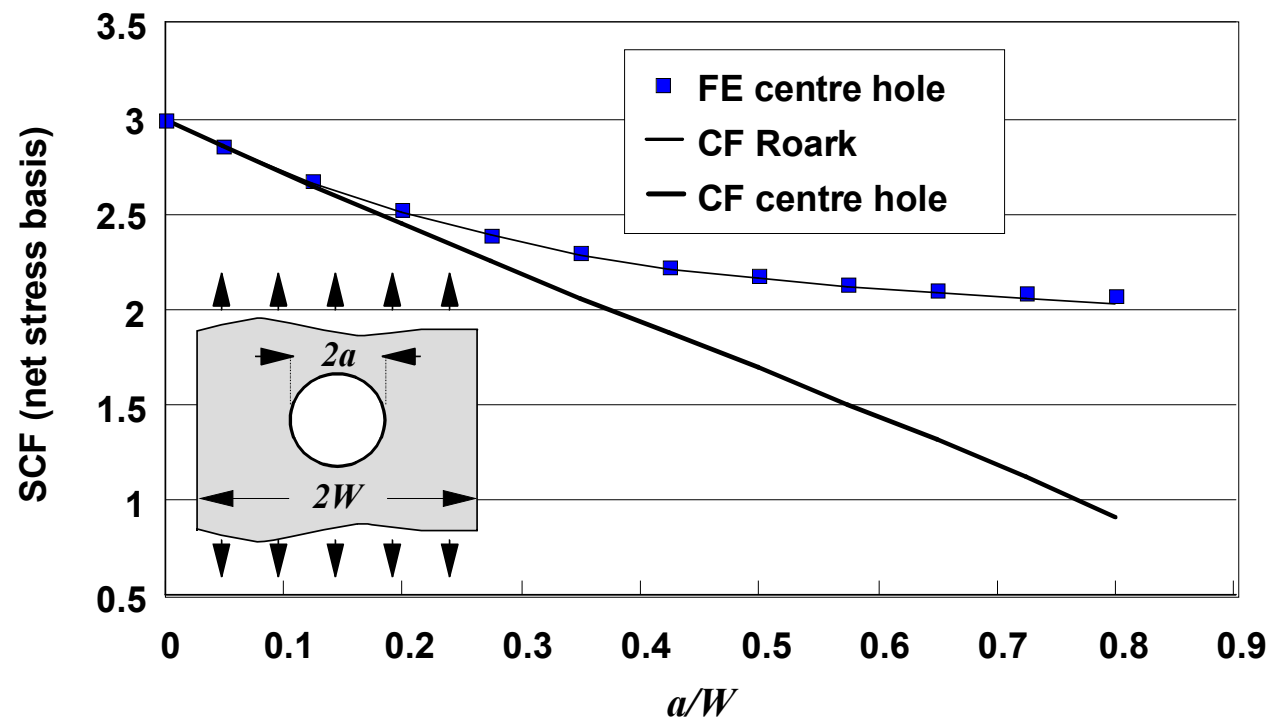

Central hole in finite width plate

Comparison of SIF-based estimate with FE determination

Figure 3(a)

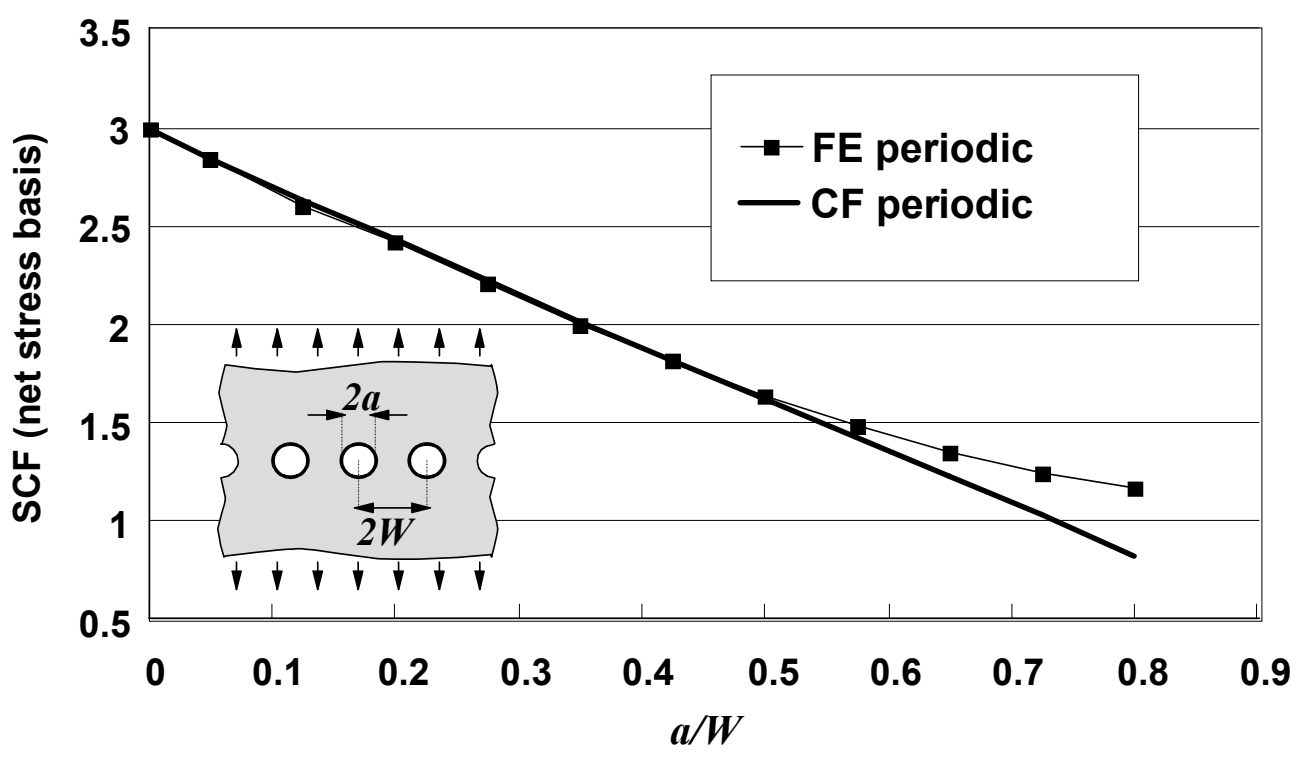

Row of equally-spaced holes transverse to load

Comparison of SIF-based estimate with FE determination

Figure 3(b) 


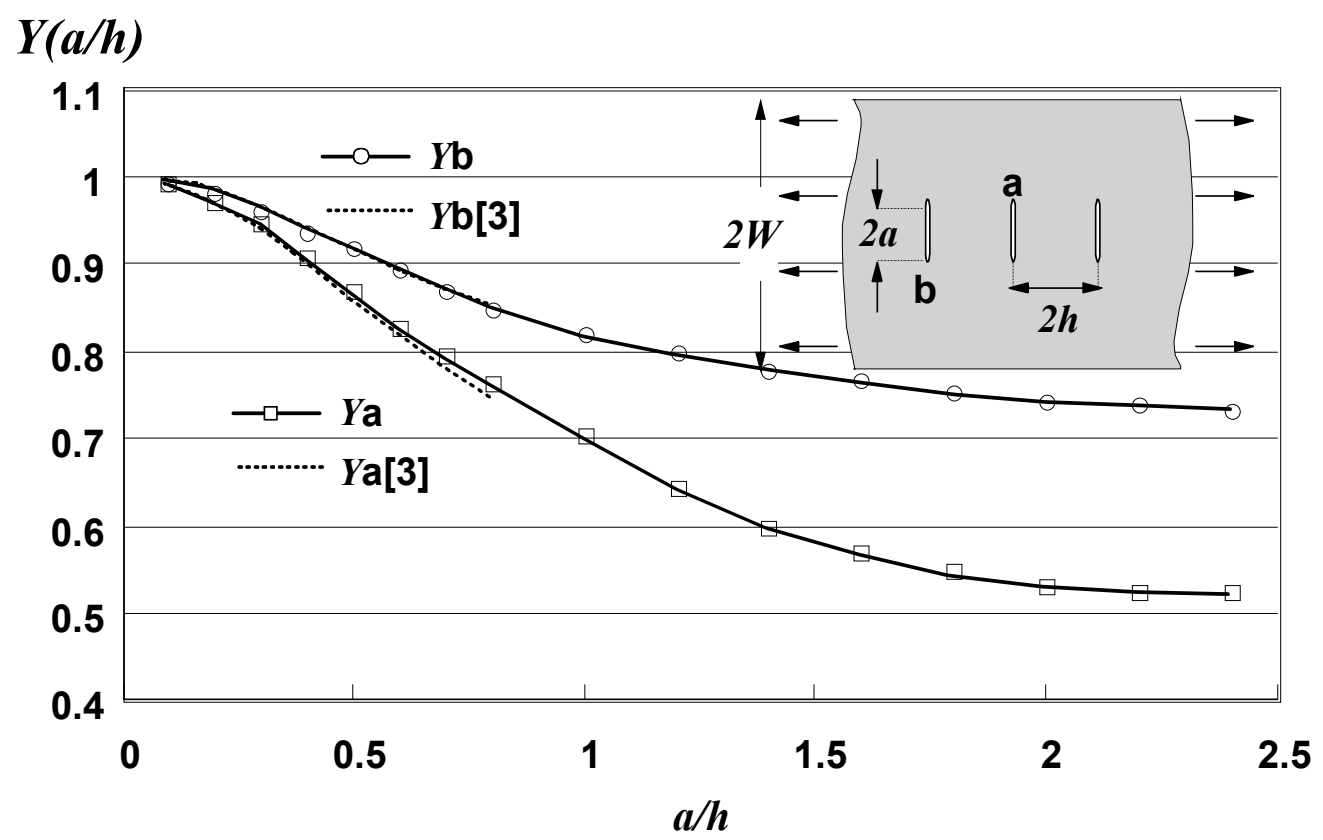

Configuration factors for 3 equally-spaced cracks in line with load

Figure 4

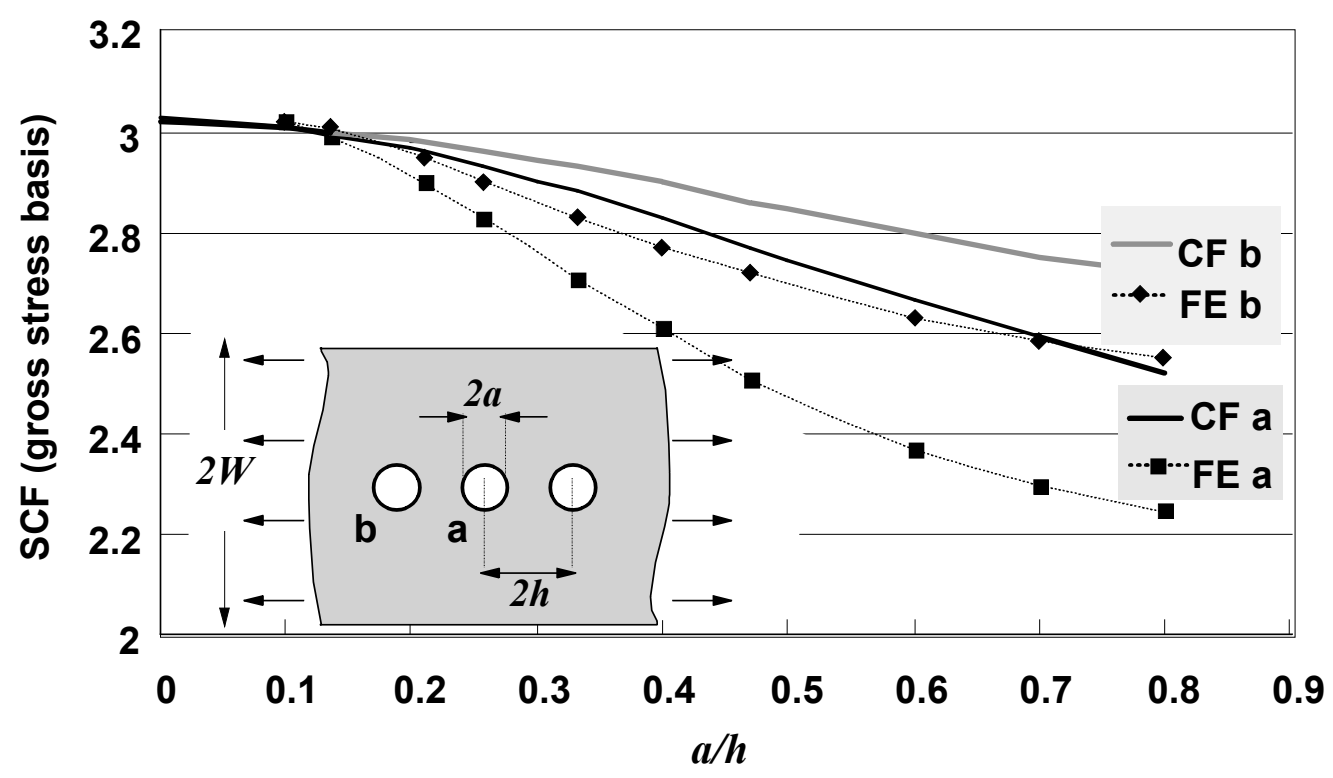

Equally-spaced holes in line with load Comparison of SIF-based estimate with FE determination

Figure 5 


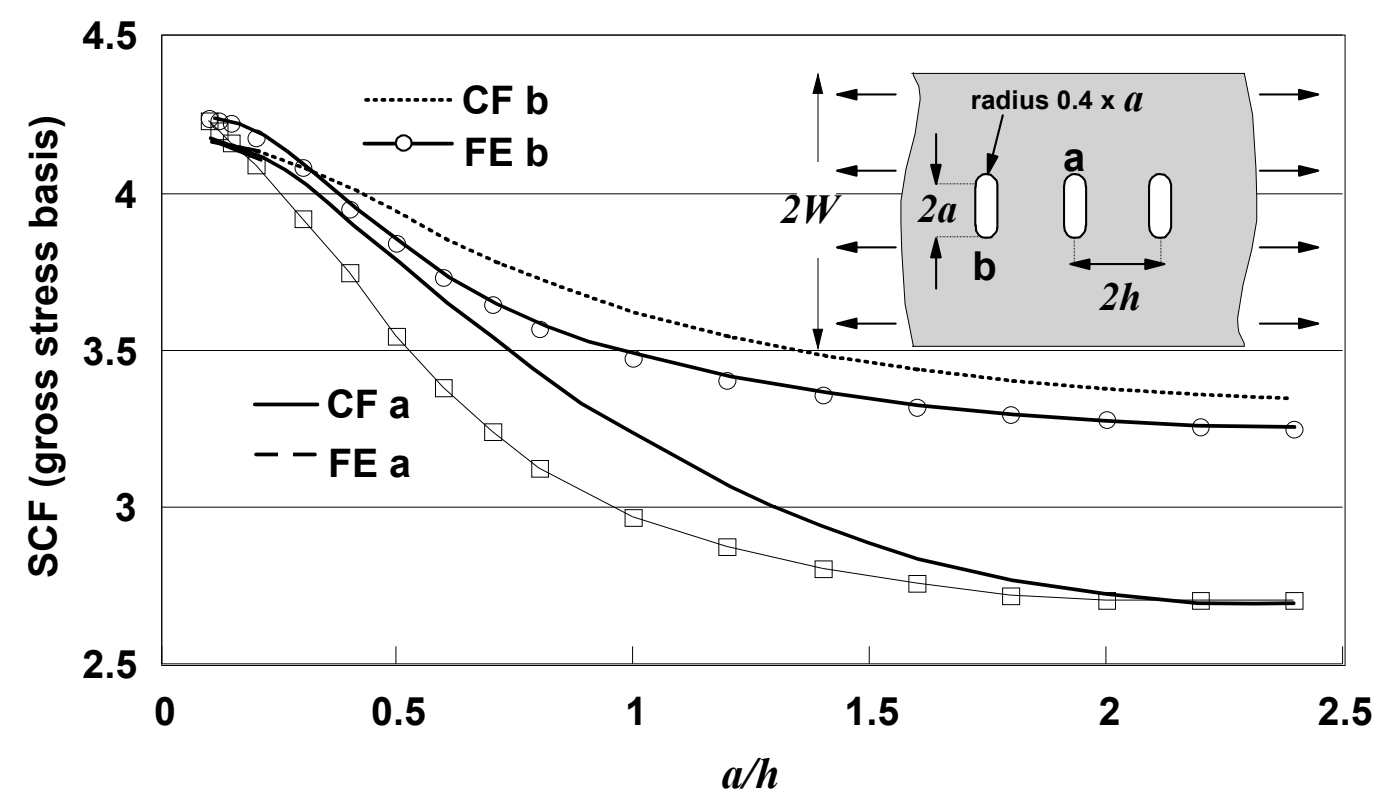

Equally-spaced slots in line with load

Comparison of SIF-based estimate with FE determination

Figure 6
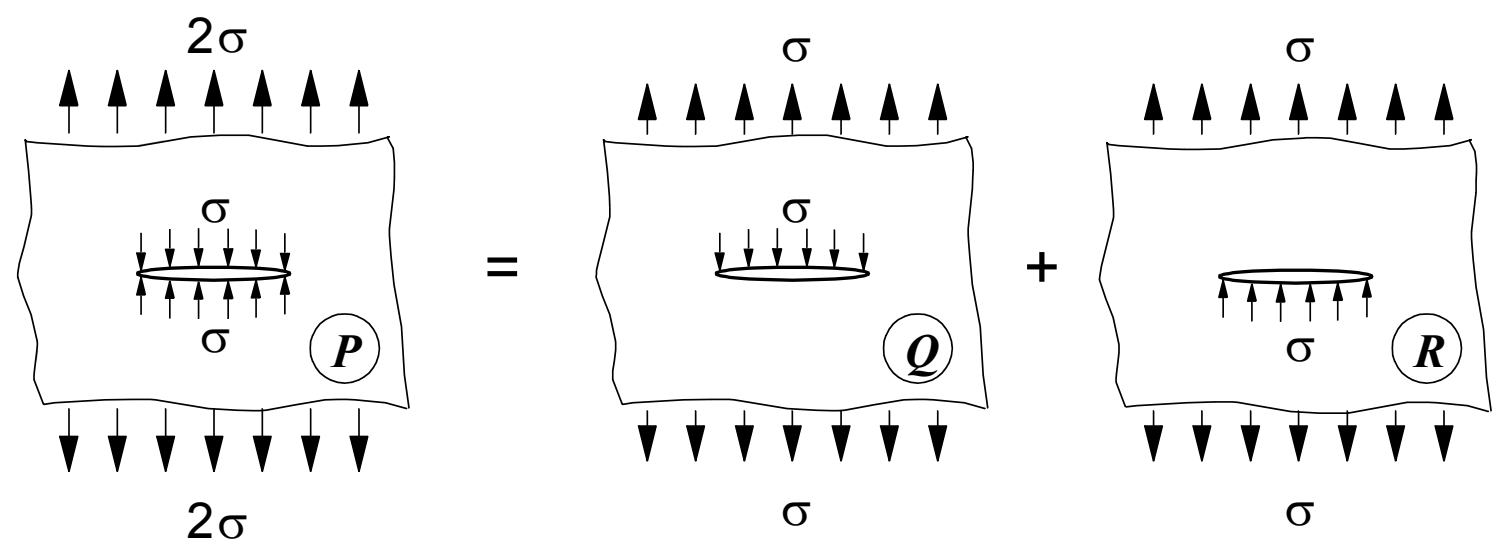

Superposition of solutions

Figure 7(a) 


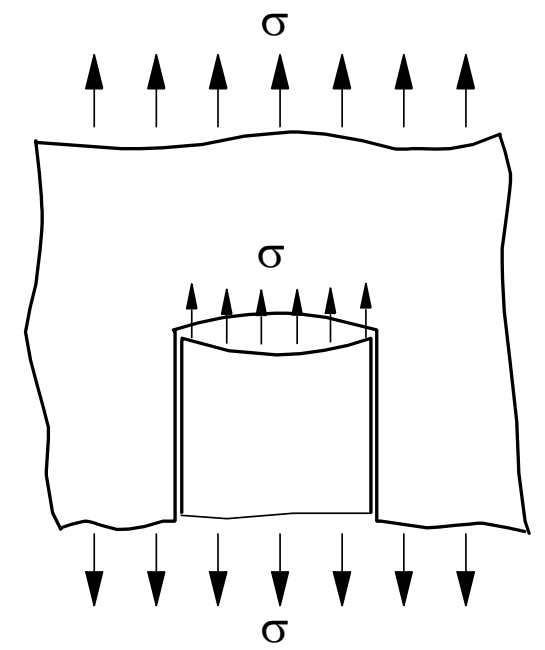

(b)

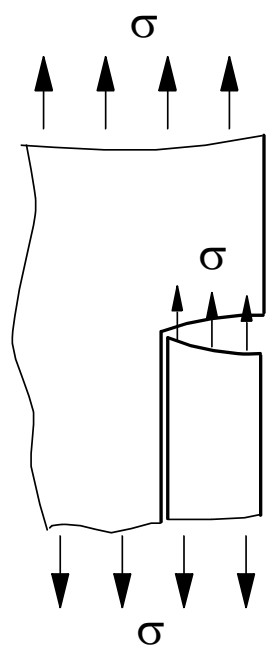

(c)

Generation of infinitely-long internal cut-out and external step

Figure $7(\mathrm{~b})$ and (c)

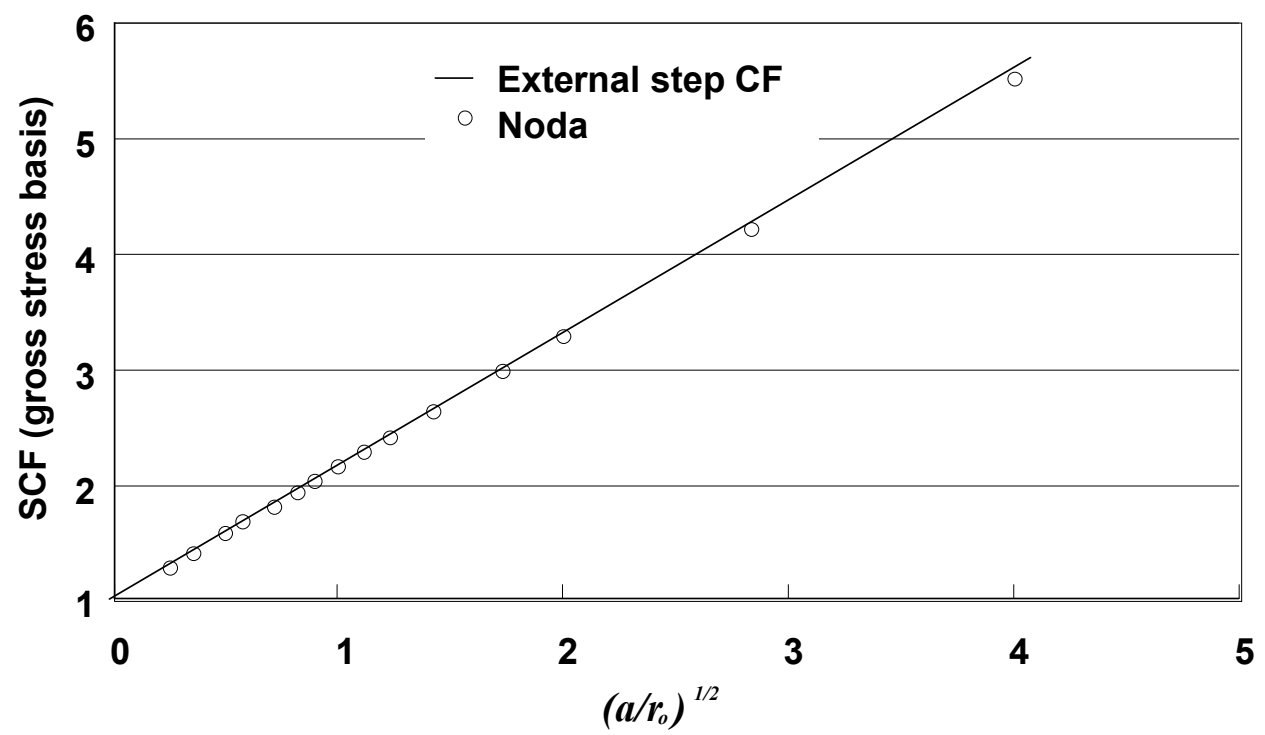

Closed form SCF (equation 5) for external, radiused step in semi-infinite plate

Figure 8 


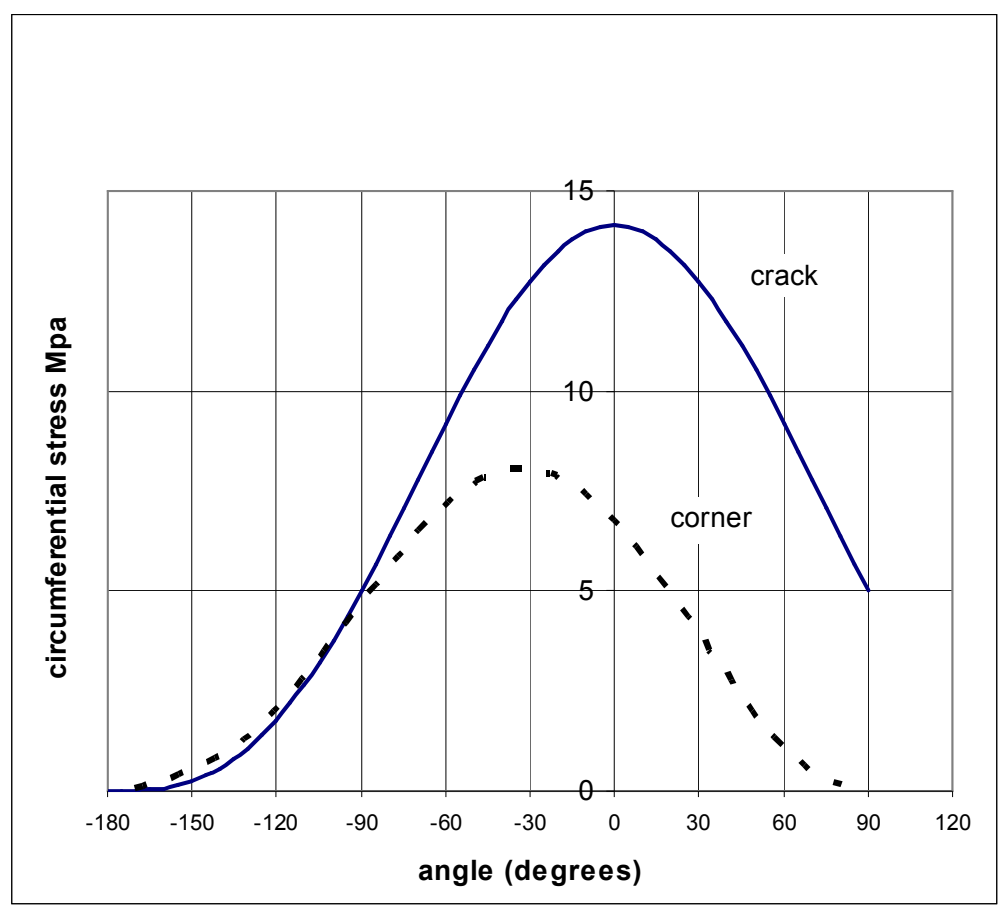

Comparison of crack/corner circumferential stress in singular region at specific radius Figure 9
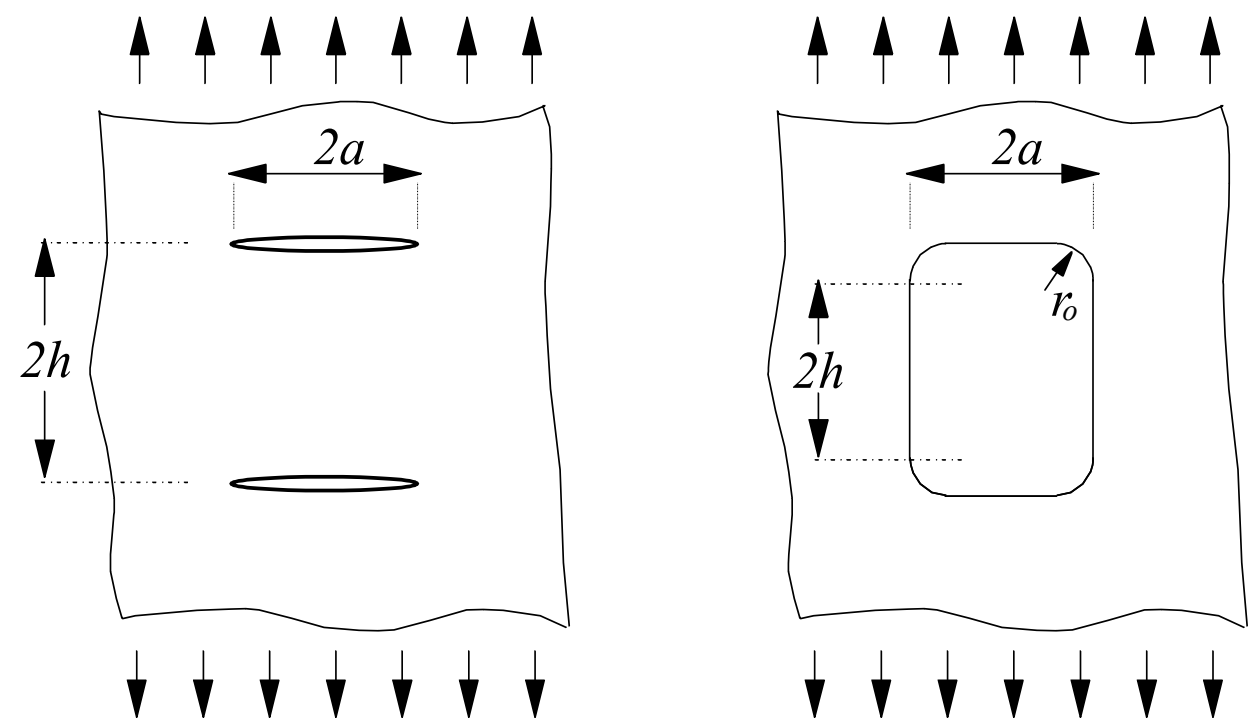

Dimensional correspondence between crack spacing and cut-out length

Figure 10 

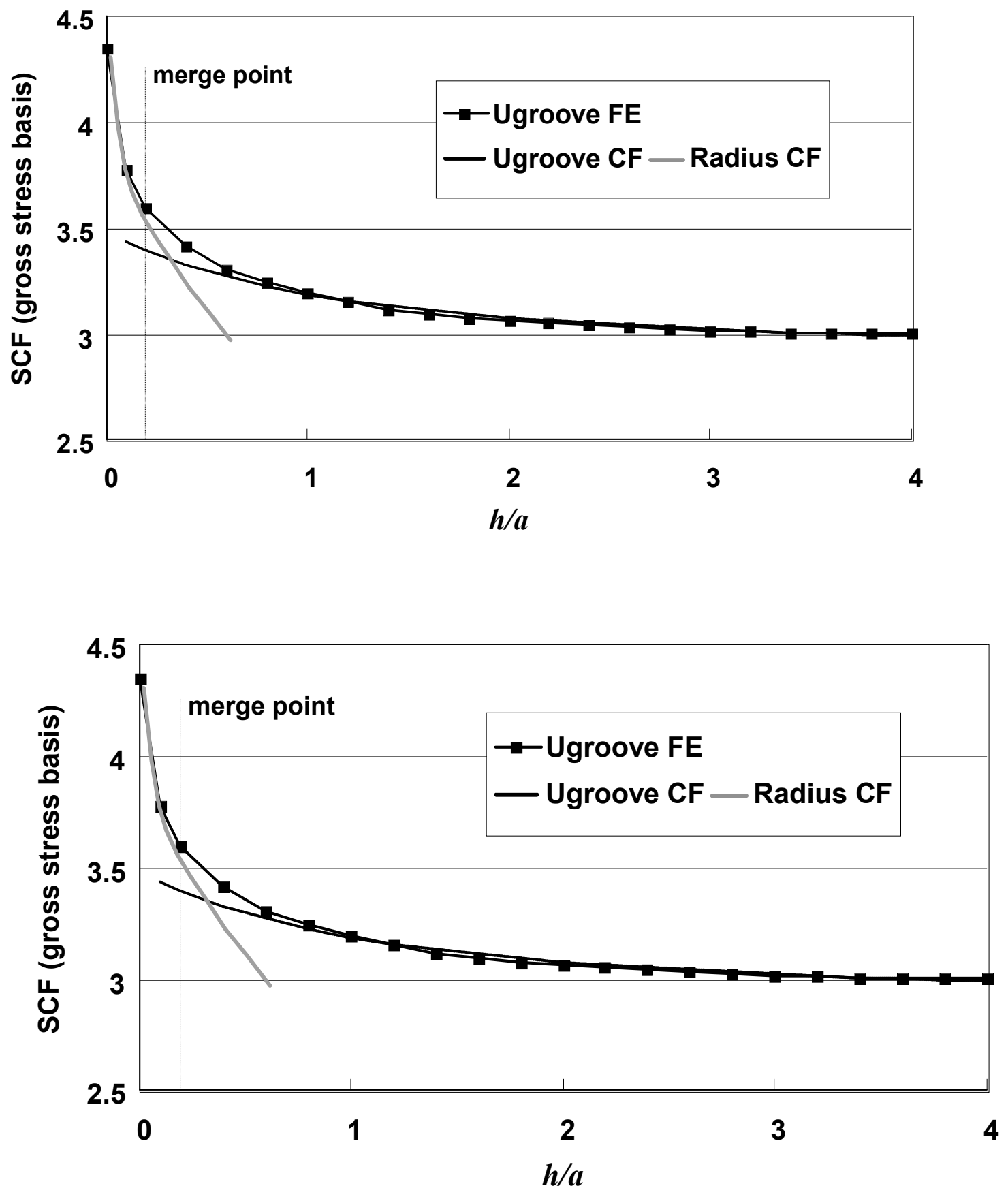

Corner-rounded rectangular cutouts and U-grooves

Comparison of SIF-based estimates with FE determination

Figure 11 
